\title{
Comorbidities in Patients with COVID-19 and Their Impact on the Severity of the Disease
}

\author{
Shahid Bashir ${ }^{1}$ Sadaf Moneeba ${ }^{2}$ Alaa Alghamdi ${ }^{3}$ \\ Hadeel Anan ${ }^{1}$ Imdad Kaleem ${ }^{4}$ \\ ${ }^{1}$ Neuroscience Center, King Fahad Specialist Hospital Dammam, \\ Dammam, Saudi Arabia \\ 2Department of Bioinformatics and Biotechnology, International \\ Islamic University Islamabad, Islamabad, Pakistan \\ ${ }^{3}$ King Fahad University Hospital, Imam Abdulrahman Bin Faisal \\ University, Dammam, Saudi Arabia \\ ${ }^{4}$ Department of Bioinformatics and Biosciences, COMSATS \\ University (CUI), Islamabad, Pakistan \\ J Health Allied Sci ${ }^{\mathrm{NU}}: 2021 ; 11: 1-7$
}

\begin{abstract}
Address for correspondence Shahid Bashir, PhD, Neuroscience Centre, King Fahad Specialist Hospital Dammam, Dammam 31444, Saudi Arabia (e-mail: shahidbpk13@gmail.com).
\end{abstract}

\begin{abstract}
Keywords

- COVID-19

- morbidity

- comorbidities

- pandemic
\end{abstract}

Infection with COVID-19 is associated with significant morbidity, especially in patients with chronic medical conditions. At least one-fifth of cases require supportive care in intensive care units, which have limited availability in most developing countries. A literature search was conducted on PubMed, Medline, Scopus, Embase, and Google Scholar to find articles published by May 7, 2020 on the role of comorbidities in patients with COVID-19 and the impact of comorbidities on the disease. This review highlighted that patients with comorbidities are more likely to experience severe disease than those with no other conditions; that is, comorbidities correlated with greater disease severity in patients with COVID-19. Proper screening of COVID-19 patients should include careful inquiries into their medical history; this will help healthcare providers identify patients who are more likely to develop serious disease or experience adverse outcomes. Better protection should also be given to patients with COVID-19 and comorbidities upon confirmation of the diagnosis. This literature review showed that the comorbidities most often associated with more severe cases of COVID-19 are hypertension, cardiovascular disease, and diabetes. Individuals with these comorbidities should adopt restrictive measures to prevent exposure to COVID-19, given their higher risk of severe disease.

\section{Introduction}

The 2019 coronavirus (COVID-19) originated in Wuhan, China, in late December 2019. Since then, the spread of this illness has continued to endanger global health while severely limiting everyday activities. The first reports of the disease stated that a cluster of patients in Wuhan were suffering from pneumonia of unknown cause. ${ }^{1}$ The cause was soon recognized as a novel coronavirus, which was first named 2019-nCov and then renamed Severe Acute Respiratory Syndrome Coronavirus 2 (SARS-CoV-2, or COVID-19) by the Coronavirus Study Group of the International Committee on the Taxonomy of Viruses. ${ }^{2}$ COVID-19 has since impacted published online

October 16, 2020
Dol https://doi.org/

$10.1055 / \mathrm{s}-0040-1718848$ ISSN 2582-4287. the entire world, with the total number of current cases surpassing 19.8 million and a total death toll of over $729 \mathrm{~K}$ individuals worldwide. ${ }^{3}$

Recent data from around the world confirm that individuals with major comorbidities, including cardiovascular disease (CVD), hypertension (HTN), diabetes, chronic obstructive pulmonary disease (COPD), and malignancies seem to be at higher risk of severe complications related to COVID-19 than those without these conditions. ${ }^{4}$

Hospitaladmission ratesfor patients with COVID-19increase further with polymorbidity, which can lead to case fatality rates as high as $14 \%$ and may require hospitalization and admission to intensive care units (ICUs). 5
This is an open access article published by Thieme under the terms of the Creative Commons Attribution-NonDerivative-NonCommercial-License, permitting copying and reproduction so long as the original work is given appropriate credit. Contents may not be used for commercial purposes, or adapted, remixed, transformed or built upon. (https://creativecommons.org/licenses/by-nc-nd/4.0/).

Thieme Medical and Scientific Publishers Pvt. Ltd. A-12, 2nd Floor, Sector 2, Noida-201301 UP, India 
One recent study found that individuals with chronic underlying illnesses have a higher risk of severe outcomes from COVID-19 than individuals without any comorbidities. ${ }^{6}$

In this review, we discuss the top comorbidities worldwide (CVD, diabetes, COPD, cancer, chronic kidney disease (CKD), smoking, and obesity) observed in patients with COVID-19; we also investigate the associations of these comorbidities with negative outcomes from COVID-19. Given the tremendous health-related and economic burdens imposed by the COVID19 pandemic, a thorough evaluation of the associations and prevalence of comorbidities in COVID-19 patients is needed to understand the role of comorbidities in this global pandemic.

This review identifies vulnerable patient populations who are at increased risk of severe COVID-19 complications. It also informs clinicians, policymakers, and researchers of new strategies and policies that have been developed to mitigate the effects of the COVID-19 pandemic.

\section{Methods}

A search strategy was designed to retrieve all relevant articles. The search terms "COVID-19 AND comorbidity" and "COVID-19 AND comorbidities, clinical characteristics, epidemiology" were used. To avoid missing any relevant literature, we performed additional searches using the reference lists of the included studies. The databases PubMed, MEDLINE, EMBASE, SCOPUS, and Google Scholar were accessed to identify all relevant articles published by May 7, 2020. Articles were screened using the abstracts, and then the inclusion criteria were applied. The full texts of articles that appeared relevant to the study topic were accessed for further screening.

The inclusion criteria were as follows: Articles about comorbidities and individuals diagnosed with COVID-19 published in 2020 in English were included. The studies included datasets of patients with COVID-19 and major comorbidities, including CVD, HTN, diabetes, COPD, CKD, and malignancy. The exclusion criteria were as follows: ${ }^{1}$ Articles that did not contain appropriate or sufficient data regarding the major comorbidities listed above were excluded. ${ }^{2}$ Studies limited to pediatric patients, pregnant patients, and disease-specific studies were excluded. ${ }^{3}$ Studies not written in English were excluded, ${ }^{4}$ as were discussion summaries, abstracts, case reports, systematic reviews, editorials, and letters.

\section{COVID-19 Symptoms and Possible Outcomes}

Recent reports indicate that the clinical manifestations of COVID-19 may include a multitude of symptoms that vary in severity. The most common symptoms are fever, cough, sputum, myalgia, fatigue, and shortness of breath. Most patients present without complications. However, significant percentages of COVID-19 patients experience severe (35\%) or critical (28\%) disease.7 Critically ill patients may suffer from adverse outcomes that necessitate ICU admission, such as sepsis, acute respiratory distress syndrome (ARDS), shock, heart failure, respiratory failure, coagulopathy, acute kidney injury, and even death. ${ }^{7}$ Everyone is susceptible to this virus, but the elderly and those with underlying diseases have a higher risk of adverse outcomes. $^{8}$

\section{The Prevalence of Comorbidities in COVID-19 Patients}

A nationwide analysis of the prevalence of comorbidities in patients with COVID-19 in China reports that almost 50\% of patients hospitalized due to COVID-19 had at least one of the following comorbidities: diabetes, HTN, CVD, or cerebrovascular disease. ${ }^{9}$ This is supported by a study showing that severely afflicted patients had higher rates of HTN, diabetes, and coronary heart disease. Moreover, COVID-19 patients with concomitant CVD have a higher risk of mortality.$^{10}$ Another comparative study in Wuhan revealed that patients who were suffering from severe illness had a higher prevalence of comorbid conditions than those with a non-severe course. This suggests that comorbidities may increase the risk that an individual will present with a severe case of COVID-19. ${ }^{8}$

In our meta-analysis of the prevalence of comorbidities in hospitalized COVID-19 patients, HTN was the most common comorbidity, occurring in $16 \%$ of hospitalized patients, followed by CVD, occurring in around $12 \%$. Diabetes mellitus occurred in $7.8 \%$ of cases, and COPD and CKD occurred in $0.95 \%$ and $0.83 \%$, respectively. ${ }^{11}$ Deficits in spirometric testing (a test of lung functionality) and a lack of awareness due to lack of careful observation played important roles in the diagnosis of respiratory disorders in this study. ${ }^{12,13}$

Early in an epidemic, the prevalence of comorbidities might reflect transmission dynamics within certain age groups; it may also affect hospital admission policies and case detection.

\section{The Impact of Comorbidities on the Severity of COVID-19}

Healthcare providers must pay careful attention to the treatment of original comorbidities in COVID-19 patients, especially those with pneumonia and particularly when those comorbidities include diabetes, CVD, COPD, CKD, HTN, or respiratory system disorders. These guidelines are even more important for older patients with serious comorbidities. It has been observed that COVID-19 is involved in the impairment of various organs and systems, including the kidneys, heart, liver, immune system, and the blood vessel system. ${ }^{14-16}$ Patients with COVID-19 may die due to ARDS, multiple organ failure, heart and renal failure, shock, or cardiac arrhythmia. ${ }^{17}$ For this reason, the treatment of patients with COVID-19 must include protection against and prevention of problems in multiple organs.

\section{Diabetes}

Patients with COVID-19 pneumonia and concurrent diabetes have been reported to have a more severe course than those without diabetes. ${ }^{18}$ Severity is evaluated in terms of organ damage, inflammatory factors, or hypercoagulability. These patients are also more likely to progress to a worse prognosis. Therefore, diabetes might be considered a risk factor for a poor outcome in patients with COVID-19 pneumonia. ${ }^{18}$ Furthermore, endocrine diseases, such as diabetes, are common in patients diagnosed with COVID-19, even apart from the likelihood of circulatory and endocrine comorbidities. One study estimated that the 
prevalence of diabetes among patients with COVID-19 was 7.87\% (95\% CI: 6.57\%-9.28\%). ${ }^{11}$

\section{Hypertension (HTN)}

The current literature suggests that HTN may be associated with an up to 2.5-fold increase in the risk of severe COVID-19, especially among older individuals. HTN is also associated with higher mortality due to COVID-19.13

The present meta-analysis finds that HTN occurs in approximately $17 \%$ of patients hospitalized with COVID-19. ${ }^{19}$ Another study found that HTN is the most prevalent underlying disease in hospitalized COVID-19 cases, occurring in 16\% of such cases (95\%: CI: 10.15-23.65\%). ${ }^{11}$

\section{Chronic Obstructive Pulmonary Disease (COPD)}

COPD is associated with over a five-fold increase in the risk of severe COVID-19 infection. ${ }^{14}$ A recent study suggested that, compared to patients with no comorbidities, patients with severe cases of COVID-19 and COPD had the highest mortality risk (OR 1.49, 95\% CI: 1.10-2.01). ${ }^{19}$ Another study found that COPD occurred in $0.95 \%$ of hospitalized COVID-19 patients (95\% CI: 0.43-1.61\%). ${ }^{11}$

Furthermore, ongoing COPD and a history of smoking contribute to more severe cases and poorer outcomes in patients with COVID-19. SARS-CoV-2 initially occupies the pulmonary alveolar epithelial cells. ${ }^{20}$ However, most infections are believed to cause only mild symptoms; ARDS is rare but can lead to multi-organ dysfunction. ${ }^{21}$

The effects of COPD and of a history of smoking on the course of a COVID-19 infection and on the disease's clinical manifestation are, however, still uncertain. ${ }^{22}$

\section{Cardiovascular Disease (CVD)}

Patients with previous CVDs are more likely to experience a severe course of COVID-19. COVID-19 may also expedite cardiac damage through multiple mechanisms, further worsening the patient's prognosis. ${ }^{23}$

One study suggested that, compared to patients with no comorbidities, patients with severe cases of COVID-19 and with CVD have a higher risk of morbidity (OR 2.92, 95\% CI: 1.76-4.86). ${ }^{24}$ In the present analysis, eight studies were evaluated to estimate the pooled prevalence of CVD in COVID-19 patients; CVD occurred in approximately 5\% of hospitalized COVID-19 cases. $^{11}$

\section{Malignancy (Malignant Tumor)}

Immunocompromised people with a history of cancer are more susceptible to COVID-19 due to anti-cancer treatments and malignancies. Similarly, healthy individuals are less susceptible to the infection than those suffering from gastro-intestinal (GI) cancer.

In a recent nationwide analysis in China, ${ }^{25} 18$ of 1,590 (1\%) COVID-19 cases had a history of cancer. Of those 18 cases, three had a history of colorectal cancer, including one colorectal carcinoma, one colonic tubular adenocarcinoma, and one rectal carcinoma. ${ }^{26}$

A recent study showed indicated that the prevalence of malignancy among hospitalized COVID-19 patients is approximately $0.92 \%$ (95\% CI: $0.56-1.34 \%) .{ }^{11}$

\section{Chronic Kidney Disease (CKD)}

A previous study found that approximately $13 \%$ of patients with COVID-19 had underlying kidney disease. ${ }^{11}$ Similarly, during hospitalization for COVID-19, up to $40 \%$ had signs of abnormal kidney function, and $5.1 \%$ had acute kidney injury (AKI). A dose-dependent association was observed between COVID-19 morbidity risk and the stage of AKI; patients with stage three AKI had a four-fold increased risk of mortality. Furthermore, a major complication of COVID-19 is a substantial morbidity risk increase by a kidney disease. $^{27}$

The main symptoms of severe cases of COVID-19 are acute respiratory failure and alveolar impairment; $2^{4}$ the involvement of other organs needs to be explored. Subsequently, the virus accumulates in the kidneys, destroying resident renal cells, and it might enter the blood after causing a lung infection. Using real-time polymerase chain reaction analysis, COVID-19 RNA has been identified in the plasma of $15 \%$ of patients. ${ }^{13}$ It is reported that $6.7 \%$ of SARS patients presented with AKI, and the mortality rate among SARS patients with AKI was $91.7 \%{ }^{28}$ Consequently, there is an urgent need to understand how the kidney is affected by COVID-19.

A study that used Kaplan-Meier statistics found that patients with kidney disease had a considerably higher risk of hospitalized death after adjusting for disease severity, age, gender, leukocyte count, and other comorbidities. ${ }^{27}$ Therefore, the prevalence of kidney disease and the development of AKI in patients with COVID-19 is high during hospitalization and is associated with in-hospital mortality. ${ }^{27}$ This means that physicians must be aware of kidney disease in patients with severe COVID-19. ${ }^{27}$

According to our pooled analysis of hospitalized patients with COVID-19, the prevalence of kidney disease is almost $0.83 \%$ (95\% CI: $0.37 \%-1.43 \%){ }^{11}$

\section{Smoking}

Our analysis shows that, of patients hospitalized with COVID-19, 7.63\% have a history of smoking. However, the six studies included in this analysis show significant heterogeneity $(\mathrm{I} 2=90.19 \%, p<0.001) .{ }^{26}$

The traditional interpretation of this finding is that the smokers are more susceptible to respiratory viruses and that smoking can up-regulate the angiotensin-converting enzyme-2 (ACE2) receptor, which is the receptor associated with human respiratory coronavirus NL638 and with severe or acute COVID19. Electronic smoking devices, such as e-cigarettes, IQOS, and heat-not-burn devices, are considered less harmful than traditional cigarettes, but these devices still cause respiratory damage. ACE2 could play a unique role in COVID-19 as an adhesion molecule; it is also a potential target of therapies to prevent infection. Therefore, to prioritize and facilitate investigation into the role of this molecule, data on smoking status must be recorded for all diagnosed cases of COVID-19.29

The evidence suggest that smokers' lungs are injured because such patients are more susceptible to pulmonary bacterial infections and viral infections. ${ }^{30}$ Similarly, smokers have a $34 \%$ higher chance of infection with influenza than nonsmokers. ${ }^{30}$ Han et al. found that smoking was consistently 
associated with an increased risk of hospital admission due to post-influenza infections. ${ }^{31}$

\section{Obesity}

Obese individuals may also have a higher risk of severe COVID-19. However, metabolic parameters, such as body mass index (BMI) and blood levels of insulin and glucose (COVID-19) are insufficient to measure this effect. More attention to this issue is needed to improve our understanding of the causes of COVID-19 and (equally important) our ability to care for patients affected by it. ${ }^{32}$

In one study, almost $85 \%$ of obese patients hospitalized with COVID-19 required a mechanical ventilator; $62 \%$ mortality was reported for COVID-19 patients with obesity. $^{32}$ Similarly, $64 \%$ of non-obese patients required mechanical ventilation, and the mortality rate for non-obese patients was $36 \%$ percent. $^{33}$

It has been repeatedly documented that comorbidities, such as diabetes mellitus, CVD, and HTN, are associated with a more severe course of COVID-19. So far, obesity has received less attention. ${ }^{34}$ However, it is a key risk factor for these other comorbidities and is often associated with poor metabolic health, such as dyslipidemia and insulin resistance. Obesity is also associated with a higher risk of pneumonia. Thus, anthropometric features and metabolic parameters must be evaluated to appropriately assess the risk of complications in patients with COVID-19. ${ }^{35}$

Moreover, according to data reported by a university hospital in Lille, France, of 124 COVID-19 patients, the need for invasive mechanical ventilation was associated with a BMI greater than $35 \mathrm{~kg} / \mathrm{m} 2$, independently of other comorbidities. ${ }^{32}$

\section{Mode of Action of Comorbidities that Influence the Course/Severity of COVID-19}

Perhaps chronic disorders gate the origin of the infection, specifically the attenuation of the innate immune response and the pro-inflammatory state. For example, diabetes mellitus occurs due to an accumulation of (activated) innate immune cells in the metabolic tissues. This permits the release IL-1 $\beta$ and TNF $\alpha$ (inflammatory mediators), which cause $\beta$-cell impairment and systemic insulin resistance. In addition, ${ }^{36}$ through microphage damage and lymphocyte dysfunction, metabolic diseases might decrease immune function, ${ }^{37}$ which could make individuals more susceptible to COVID-19 complications. ${ }^{38}$

In a retrospective study, Guo et al. ${ }^{39}$ analyzed clinical data from patients with a history of viral pneumonia and found that the absolute levels of CD4+ T cells, CD3+ T cells, CD3+ cells, and CD3+CD8+ T cells were considerably lower in patients who died than in those who survived. This suggests that several measures indicating inflammation were lower in patients who died. ${ }^{8}$

These comorbidities may also facilitate COVID-19 complications via multiple mechanisms, leading to poorer outcomes. For example, diabetes mellitus is known to lead to a compromised immune state by impairing macrophage and lymphocyte function.37 This in turn makes the patient more likely to experience severe illness and develop complications.

Another possible mechanism implicates ACE2 imbalance as the cause of severe disease in patients with both COVID-19 and CVD.This can beexplained by the fact that ACE2 is the mainactive peptide in the renin-angiotensin-aldosterone-system. ${ }^{29}$ By targeting angiotensin, ACE2 acts as a protective compound for the cardiovascular system; it even demonstrates protective effects against respiratory failure in some infections. ${ }^{29}$ Like SARS, SARS-CoV-2 is believed to invade the host through the cell entry receptor ACE2. ${ }^{40,41}$

SARS-CoV-2 has been shown to bind to ACE2 with high affinity. This reduces the amount of active ACE2, limiting its protective effect and aggravating CVD and COVID-19. This may explain why patients with cardiovascular comorbidities are more likely to present with a more severe disease course than those without CVD. ${ }^{42}$

Moreover, patients with diabetes and HTN who are treated with ACE inhibitors may be at an increased risk of developing COVID-19.42 This can be explicated by the fact that human pathogenic coronaviruses, like SARS-CoV-2, bind to their target cells via ACE2.43 ACE2 expression is substantially increased in patients with diabetes and HTN who are treated with ACE inhibitors. ${ }^{41}$ This increased expression of ACE2 may facilitate COVID-19 infection by increasing its cellular binding and expediting its entry into cells. ${ }^{43}$

Glycolipid metabolic disorders such as hyperlipidemia, diabetes, and atherosclerotic CVD may also play a role in increasing disease severity in COVID-19 patients. ${ }^{40-43}$ These disorders are associated with chronic inflammation and elevated cytokine levels, both of which are associated with more severe cases of COVID-19. ${ }^{42}$

Several studies have illustrated the fundamental mechanisms of these associations. Similarly, Kulscar et al. found that MERS-CoV infections lead to immune cell dysfunction, prolonged airway inflammation, and alteration expression profiles of inflammatory mediators in diabetic mice models. ${ }^{44}$

Our analysis indicates that COVID-19 may lead to immune dysregulation; this might contribute to the increased risk associated with CVD, malignancies, and bone diseases. ${ }^{45}$ Prolonged inflammation and dysregulation of the immune system may be key drivers of poor clinical results in patients with these comorbidities and COVID-19, but verification via more mechanistic studies is needed. ${ }^{46}$

Patients with coexisting comorbidities are more likely to have poorer baseline health. Therefore, patients with more than two comorbidities have an increased risk of a poor prognosis. ${ }^{9,10}$ This means that, when determining the prognosis for COVID-19 patients, the number and type of comorbidities must be considered. ${ }^{9}$ Disease severity partially depends on the ratio of comorbidities, and proper triage requires a careful inquiry into a patient's medical history, as this will help healthcare providers identify patients who are more likely to develop a severe case. Early diagnosis of potential problems will better protect patients with COVID-19 and other comorbidities.

However, underreporting of COVID-19 cases may result in an overestimation of the strength of these associations and 
thus of severe outcomes; this is consistent with existing literature. ${ }^{13}$ Proper documentation of all patients at the time of hospital admission must include a complete patient history. ${ }^{48}$

\section{COVID-19 is Associated with Significant Morbidity}

In patients hospitalized with COVID-19, the casualty rate is higher than ten percent, regardless of treatment. In most cases, COVID-19 presents similarly to other respiratory viral infectious agents (pathogens), with symptoms such as cough, dyspnea, and fever. Unique elements include the rapid progression to ARDS and leukopenia observed in severe cases of COVID-19. More research is needed to identify the natural host of SARS-CoV-2 and the viral factors in fatal and severe infections. ${ }^{49}$

Hong et al. found that CVD and diabetes mellitus are significantly associated with COVID-19 complications; they also observed that diabetes did not depend on the risk factor for chronic influenza. ${ }^{38}$

Another study found that, in patients with MERS-CoV, smoking, diabetes, and cardiac disease were significantly associated with more severe illness. ${ }^{50}$

\section{MERS-CoV, Avian Influenza, and SARS-CoV}

Current literature indicates that avian influenza and SARS-CoV are more likely than MERS-CoV ${ }^{51,52}$ to lead to poor clinical outcomes. ${ }^{53,54}$

The function of the spike protein found on coronaviruses is similar to that of the hemagglutinin (HA) protein in avian influenza viruses; the rapid replication and transmission of avian influenza in compact chicken populations indicate that it is an acquirer of polybasic cleavage sites. ${ }^{55}$ Acquisition of polybasic cleavage sites in the hemagglutinin protein by recombination or insertion transforms low-pathogenicity avian influenza viruses into highly pathogenic forms; ${ }^{51}$ this process has also been detected after repeated passages in animal cell cultures. ${ }^{56}$

\section{Various Comorbidities their Association and Prognosis}

Several comorbidities are associated with poor prognoses in COVID-19 patients; these comorbidities include diabetes, respiratory diseases, HTN, malignancies, renal disease, pregnancy, and cardiac diseases. ${ }^{11,58}$ Comorbidities associated with poor outcomes in other outbreaks of severe acute respiratory diseases, such as COPD, HTN, malignancies, and diabetes, may also predispose patients with COVID-19 to more severe cases. ${ }^{54,56,59,60}$ However, the strength of this association between COVID-19 prognosis and various comorbidities is unclear as it varies in different reports ${ }^{61,62}$; for example, the association of CVD with poor clinical outcomes of SARS$\mathrm{CoV}$, MERS-CoV, or influenza remains uncertain. Apart from diabetes, no other comorbidities have been recognized as prognosticators of poor clinical outcomes in patients with MERS-CoV. ${ }^{63}$

\section{Conclusion}

The COVID-19 pandemic has severely damaged the health, social, and economic sectors around the world. The global impact of this pandemic highlights the importance of studies that may identify the risk factors associated with poor outcomes from COVID-19. Identifying the comorbidities that implicate poorer outcomes are a key part of the global effort to characterize, evaluate, and manage this pandemic. More evidence is needed to clarify and define vulnerable groups (currently believed to be individuals over 65 and those with one or more preexisting comorbid conditions). This literature review has explored the global prevalence of HTN, CVD, and diabetes as comorbidities among individuals with severe cases of COVID-19. Identifying these comorbidities will help healthcare providers identify COVID-19 patients with a poor prognosis, which can therefore allow hospital personnel to apply interventions that may mitigate complications and improve overall outcomes. Additional studies with larger samples are needed to further investigate these associations.

The information on comorbidities that increase the risk of severe COVID-19 is constantly changing, and periodic updates may aid our ability to combat this global pandemic. This review has highlighted the importance of identifying vulnerable patient populations with comorbidities that may lead to complications of COVID-19. Due to this high risk, individuals with one or more underlying comorbidities should take extra precautions to avoid close contact with members of their communities, particularly in areas with high infection rates. Although researchers and major pharmaceutical companies around the world are racing to develop anti-viral therapies and a vaccination, focused efforts are currently needed to protect these vulnerable groups.

\section{Conflict of Interest}

None declared.

\section{References}

1 Hui DS, I Azhar E, Madani TA, et al. The continuing 2019-nCoV epidemic threat of novel coronaviruses to global health - The latest 2019 novel coronavirus outbreak in Wuhan, China. Int J Infect Dis 2020;91:264-26610.1016/j.ijid.2020.01.009

$2 \mathrm{Wu} \mathrm{Y,} \mathrm{Ho} \mathrm{W,} \mathrm{Huang} \mathrm{Y,} \mathrm{et} \mathrm{al.} \mathrm{SARS-CoV-2} \mathrm{is} \mathrm{an} \mathrm{appropriate}$ name for the new coronavirus. Lancet 2020;395(10228): 949-950

3 Worldometer. Available at: https://www.worldometers. info/coronavirus/?utm_campaign=homeAdvegas1? Accessed October 5,2020

4 Preliminary estimates of the prevalence of selected underlying health conditions among patients with coronavirus disease 2019 MMWR Morb Mortal Wkly Rep. CDC; February 12-March 282020

5 Coronavirus disease. 2019 (COVID-19) in the EU/EEA and the UK - eighth update. European Center for Disease Prevention and Control. 2020

6 Bommer W. CDC offers guidance to patients with chronic disease 'living with uncertainty' during COVID-19. Healio Rheumatology; 2020

7 Zhou F, Yu T, Du R, et al. Clinical course and risk factors for mortality of adult inpatients with COVID-19 in Wuhan, China: a retrospective cohort study. Lancet 2020;395(10229) :1054-1062

8 Yang J, Zheng Y, Gou X, et al. Prevalence of comorbidities in the novel Wuhan coronavirus (COVID-19) infection: a systematic review and meta-analysis. Int J Infect Dis 2020;94:91-95

9 Guan W-J, Liang W-H, Zhao Y, et al; China Medical Treatment Expert Group for COVID-19. Comorbidity and its impact on 
1590 patients with COVID-19 in China: a nationwide analysis. Eur Respir J 2020;55(5):2000547

10 Peng YD, Meng K, Guan HQ et al. [Clinical characteristics and outcomes of 112 cardiovascular disease patients infected by 2019-nCoV]. Zhonghua Xin Xue Guan Bing Za Zhi. 2020;48(6):450-455

11 Emami A, Javanmardi F, Pirbonyeh N, Akbari A. Prevalence of underlying diseases in hospitalized patients with COVID-19: a systematic review and meta-analysis. Arch Acad Emerg Med 2020;8(1):e35

12 Fang L, Gao P, Bao H, et al. Chronic obstructive pulmonary disease in China: a nationwide prevalence study. Lancet Respir Med 2018;6(6):421-430

13 Huang C, Wang Y, Li X, et al. Clinical features of patients infected with 2019 novel coronavirus in Wuhan, China. Lancet 2020;395(10223) :497-506

14 Wang D, Hu B, Hu C, et al. Clinical characteristics of 138 hospitalized patients with 2019 novel coronavirus-infected pneumonia in Wuhan, China. JAMA 2020;323(11): 1061-1069

15 Zhang JJ, Dong X, Cao YY, et al. Clinical characteristics of 140 patients infected with SARS-CoV-2 in Wuhan, China. Allergy 2020;75(7):1730-1741

16 Chen N, Zhou M, Dong X, et al. Epidemiological and clinical characteristics of 99 cases of 2019 novel coronavirus pneumonia in Wuhan, China: a descriptive study. Lancet 2020;395(10223): 507-513

17 Epidemiology Working Group for NCIP Epidemic Response, Chinese Center for Disease Control and Prevention. [The epidemiological characteristics of an outbreak of 2019 novel coronavirus diseases (COVID-19) in China]. Zhonghua Liu Xing Bing Xue Za Zhi. 2020;41(2):145-151

18 Guo W, Li M, Dong Y, et al. Diabetes is a risk factor for the progression and prognosis of COVID-19. Diabetes Metab Res Rev 2020. Doi: 10.1002/dmrr.3319

19 S. Hu, R. Gao, L. Liu, M. Zhu, W. Wang, Y. Wang, et al. Summary of the 2018 report on cardiovascular diseases in China Chin Circ J, 34 (2019), p. 209

20 Li YC, Bai WZ, Hashikawa T. The neuroinvasive potential of SARS-CoV2 may play a role in the respiratory failure of COVID-19 patients. J Med Virol 2020;92(6):552-555

21 Cao Y, Liu X, Xiong L, Cai K. Imaging and clinical features of patients with 2019 novel coronavirus SARS-CoV-2: A systematic review and meta-analysis. J Med Virol 2020

22 Zhao Q Meng M, Kumar R, et al. The impact of COPD and smoking history on the severity of COVID-19: A systemic review and meta-analysis. J Med Virol 2020

23 Li B, Yang J, Zhao F, et al. Prevalence and impact of cardiovascular metabolic diseases on COVID-19 in China. Clin Res Cardiol 2020;109(5):531-538

24 Mertz D, Kim TH, Johnstone J, et al. Populations at risk for severe or complicated influenza illness: systematic review and meta-analysis. BMJ 2013;347:f5061

25 Mao R, Liang J, Shen J, et al; Chinese Society of IBD, Chinese Elite IBD Union; Chinese IBD Quality Care Evaluation Center Committee. Implications of COVID-19 for patients with pre-existing digestive diseases. Lancet Gastroenterol Hepatol 2020;5(5):425-427

26 Liang W, Guan W, Chen R, et al. Cancer patients in SARS-CoV-2 infection: a nationwide analysis in China. Lancet Oncol 2020;21(3):335-337

27 Cheng Y, Luo R, Wang K, et al. Kidney disease is associated with in-hospital death of patients with COVID-19. Kidney Int 2020;97(5):829-838

28 Chu KH, Tsang WK, Tang CS, et al. Acute renal impairment in coronavirus-associated severe acute respiratory syndrome. Kidney Int 2005;67(2):698-705
29 Brake SJ, Barnsley K, Lu W, McAlinden KD, Eapen MS, Sohal SS. Smoking upregulates angiotensin-converting enzyme-2 receptor: a potential adhesion site for novel coronavirus SARS-CoV-2 (Covid-19) J Clin Med 2020;9(3):841

30 Lawrence H, Hunter A, Murray R, Lim WS, McKeever T. Cigarette smoking and the occurrence of influenza - Systematic review. J Infect 2019;79(5):401-406

31 Han L, Ran J, Mak YW, et al. Smoking and Influenza-associated Morbidity and Mortality: A Systematic Review and Meta-analysis. Epidemiology 2019;30(3):405-417

32 Simonnet A, Chetboun M, Poissy J, et al; LICORN and the Lille COVID-19 and Obesity study group. High prevalence of obesity in severe acute respiratory syndrome coronavirus-2 (SARS-CoV-2) requiring invasive mechanical ventilation. Obesity (Silver Spring) 2020;28(7):1195-1199

33 Bhatraju PK, Ghassemieh BJ, Nichols M, et al. Covid-19 in critically ill patients in the Seattle region-case series. N Engl J Med 2020;382(21):2012-2022

34 Petrilli CM, Jones SA, Yang J, et al. Factors associated with hospitalization and critical illness among 4,103 patients with COVID-19 disease in New York City. BMJ 2020;369:m1966

35 Stefan N, Birkenfeld AL, Schulze MB, Ludwig DS. Obesity and impaired metabolic health in patients with COVID-19. Nat Rev Endocrinol 2020;16(7):341-342

36 Odegaard JI, Chawla A. Connecting type 1 and type 2 diabetes through innate immunity. Cold Spring Harb Perspect Med 2012;2(3):a007724

37 Dooley KE, Chaisson RE. Tuberculosis and diabetes mellitus: convergence of two epidemics. Lancet Infect Dis 2009;9(12):737-746

38 Hong KW, Cheong HJ, Choi WS, et al. Clinical courses and outcomes of hospitalized adult patients with seasonal influenza in Korea, 2011-2012: Hospital-based Influenza Morbidity \& Mortality (HIMM) surveillance. J Infect Chemother 2014;20(1):9-14

39 Guo L, Wei D, Zhang X, et al. Clinical features predicting mortality risk in patients with viral pneumonia: the MuLBSTA Score. Front Microbiol 2019;10:2752

40 Zhou P, Yang X-L, Wang X-G, et al. A pneumonia outbreak associated with a new coronavirus of probable bat origin. Nature 2020;579(7798):270-273

41 Pandemic PC-19, Viruses C, Mellitus D. 2 Ranganath Muniyappa and Sriram Gubbi.

42 Chen Y, Gong X, Wang L, Guo J. Effects of hypertension, diabetes and coronary heart disease on COVID-19 diseases severity: a S ystematic review and meta-analysis [Internet]. medRxiv; 2020. Doi: 10.1101/2020.03.25.20043133

43 Fang L, Karakiulakis G, Roth M. Are patients with hypertension and diabetes mellitus at increased risk for COVID-19 infection? Lancet Respir Med 2020;8(4):e2110.1016/ s2213-2600(20)30116-8

44 Kulcsar KA, Coleman CM, Beck SE, Frieman MB. Comorbid diabetes results in immune dysregulation and enhanced disease severity following MERS-CoV infection. JCI Insight 2019; 4(20):131774

45 Moni MA, Liò P. Network-based analysis of comorbidities risk during an infection: SARS and HIV case studies. BMC Bioinformatics 2014;15(1):333

46 Naqvi AA, Shah A, Ahmad R, Ahmad N. Developing an integrated treatment pathway for a post-coronary artery bypass grating $(\mathrm{CABG})$ geriatric patient with comorbid hypertension and type 1 diabetes mellitus for treating acute hypoglycemia and electrolyte imbalance. J Pharm Bioallied Sci 2017;9(3): 216-220

47 Murphy TE, McAvay GJ, Allore HG, Stamm JA, Simonelli PF. Contributions of COPD, asthma, and ten comorbid conditions to health care utilization and patient-centered outcomes 
among US adults with obstructive airway disease. Int J Chron Obstruct Pulmon Dis 2017;12:2515-2522

$48 \mathrm{Xu} \mathrm{XW}, \mathrm{Wu} \mathrm{XX}$, Jiang XG, et al. Clinical findings in a group of patients infected with the 2019 novel coronavirus (SARS-Cov-2) outside of Wuhan, China: retrospective case series. BMJ 2020;368:m606

49 Rodriguez-Morales AJ, Cardona-Ospina JA, GutiérrezOcampo E, et al; Latin American Network of Coronavirus Disease 2019-COVID-19 Research (LANCOVID-19). Electronic address:, https://www.lancovid.org. Clinical, laboratory and imaging features of COVID-19: A systematic review and meta-analysis. Travel Med Infect Dis 2020;34:101623

50 Alraddadi BM, Watson JT, Almarashi A, et al. Risk factors for primary middle east respiratory syndrome coronavirus illness in humans, Saudi Arabia, 2014. Emerg Infect Dis 2016;22(1):49-55

51 Placzek HE, Madoff LC. Association of age and comorbidity on 2009 influenza A pandemic H1N1-related intensive care unit stay in Massachusetts. Am J Public Health 2014; 104(11):e118-e125

52 Gutiérrez-González E, Cantero-Escribano JM, Redondo-Bravo L, San Juan-Sanz I, Robustillo-Rodela A, Cendejas-Bueno E; Influenza Working Group. Effect of vaccination, comorbidities and age on mortality and severe disease associated with influenza during the season 2016-2017 in a Spanish tertiary hospital. J Infect Public Health 2019;12(4):486-491

53 Alqahtani FY, Aleanizy FS, Ali El Hadi Mohamed R, et al. Prevalence of comorbidities in cases of Middle East respiratory syndrome coronavirus: a retrospective study. Epidemiol Infect 2019;147:e35

54 Matsuyama R, Nishiura H, Kutsuna S, Hayakawa K, Ohmagari N. Clinical determinants of the severity of Middle East respiratory syndrome (MERS): a systematic review and meta-analysis. BMC Public Health 2016;16(1):1203
55 Alexander DJ, Brown IH. History of highly pathogenic avian influenza. Rev Sci Tech 2009;28(1):19-38

56 Ito T, Goto H, Yamamoto E, et al. Generation of a highly pathogenic avian influenza A virus from an avirulent field isolate by passaging in chickens. J Virol 2001;75(9):4439-4443

57 Garbati MA, Fagbo SF, Fang VJ, et al. A comparative study of clinical presentation and risk factors for adverse outcome in patients hospitalised with acute respiratory disease due to MERS coronavirus or other causes. PLoS One 2016;11(11):e0165978

58 Patel U, Malik P, Usman MS, et al. Age-adjusted risk factors associated with mortality and mechanical ventilation utilization amongst COVID-19 hospitalizations--a systematic review and meta-analysis. SN Compr Clin Med 2020;1-10. doi:10.1007/s42399-020-00476-w

59 Booth CM, Matukas LM, Tomlinson GA, et al. Clinical features and short-term outcomes of 144 patients with SARS in the greater Toronto area. JAMA 2003;289(21):2801-2809

60 Shiley KT, Nadolski G, Mickus T, Fishman NO, Lautenbach E. Differences in the epidemiological characteristics and clinical outcomes of pandemic (H1N1) 2009 influenza, compared with seasonal influenza. Infect Control Hosp Epidemiol 2010;31(7):676-682

61 Rahman A, Sarkar A. Risk Factors for Fatal Middle East Respiratory Syndrome Coronavirus Infections in Saudi Arabia: Analysis of the WHO Line List, 2013-2018. Am J Public Health 2019;109(9):1288-1293

62 Rivers CM, Majumder MS, Lofgren ET. Risks of death and severe disease in patients with Middle East respiratory syndrome coronavirus, 2012-2015. Am JEpidemiol 2016;184(6):460-464

63 Mauskopf J, Klesse M, Lee S, Herrera-Taracena G. The burden of influenza complications in different high-risk groups: a targeted literature review. J Med Econ 2013;16(2):264-277 